\title{
O elemento janela versus desempenho e conforto térmico: uma sistematização de lite- ratura
}

The window element versus thermal performance and comfort: a systematization of literature

Edna Aparecida Nico Rodrigues*, Victor Moura Bussolotti**

*Doutora em Arquitetura e Urbanismo. Universidade Federal do Espírito Santo. ORCID: 0000-0002-0905-1723
**Mestre em Arquitetura e Urbanismo. Universidade Federal do Espírito Santo. ORCID: 0000-0002-8645-5879

Recebido: 02/04/2020

Aceito: $22 / 06 / 2020$

https://doi.org/10.37916/arq.urb.v28i.422

usjt • arq.urb • número 28 | maio-agosto de 2020

\section{Abstract}

Em 2050 o mundo terá quase 10 bilhões de pessoas e o comprometimento dos limites ecológicos será um problema. O objetivo do artigo foi identificar quais são as características da janela mais abordadas na produção científica internacional, com ênfase na obtenção do desempenho energético da edificação e o conforto térmico. Foram utilizados dados bibliográficos indexados nas bases de dados Scopus, Web of Science e Science Direct. Os procedimentos metodológicos definiram os critérios para a escolha das bases de dados eletrônicas, as estratégias de pesquisa e definição dos descritores de busca e identificaram os parâmetros de relevância dos procedimentos para análise do conteúdo e sua sistematização. As informações obtidas resultaram em 744 artigos relacionados ao objeto de estudo. Para melhor organizacão dos resultados, a classificação do conteúdo pesquisado foi dividida em duas categorias: os elementos e posicionamento da janela; e os fatores que influenciam no desempenho da edificação. Os resultados mostraram que, dentre os elementos, o tipo de envidraçamento das janelas é o assunto mais estudado, já entre os fatores, o consumo de energia lidera. Tem-se a China como principal país pesquisador sobre o assunto, tendo o Brasil ficado com a $17^{a}$ posição. O ano de 2017 apresentou o maior número de publicações.

Palavras-chave: Esquadria. Desempenho. Eficiência Energética.
By 2050 the world will have almost 10 billion people and compromising ecological boundaries will be a problem. The objective of the paper was to identify the characteristics of the window most addressed in international scientific production, with an emphasis on obtaining the building's energy performance and thermal comfort. Bibliographic data indexed in the Scopus, Web of Science and Science Direct databases were used. The methodological procedures defined the criteria for choosing electronic databases, the research strategies and definition of the search descriptors and defined the parameters of relevance and the procedures for analyzing the content and its systematization. The information obtained resulted in 744 articles related to the object of study. For better organization of the results, the classification of the researched content was divided into two categories: the elements and positioning of the window; and the factors that influence building performance. The results showed that among the elements, the type of glazing of windows is the most studied subject, among the factors, energy consumption leads. China is the main research country on the subject, with Brazil taking 17th place. The year 2017 presented the largest number of publications.

Keywords: Window. Performance. Energy Efficiency. 


\section{Introdução}

$\mathbf{P}_{\text {ropoct }}$ se 10 bilhões de pessoas até 2050 , sem comprometimento dos limites ecológicos de nosso planeta e seus benefícios, são problemas e responsabilidades graves que a humanidade enfrentará. Um planeta saudável é a base necessária para o bem-estar geral e o avanço da sociedade (UNITED NATIONS, 2018; ORGANISATION FOR ECONOMIC CO-OPERATION AND DEVELOPMENT, 2017).

O ano de 2019 foi, segundo a Organização das Nações Unidas (UN, 2019), um dos anos mais quentes registrados e com cenários climáticos extremos. As mudanças climáticas geram consequências econômicas, ambientais e sociais profundas e que afetam a todos (AGOPYAN; JOHN, 2011), resultando em alterações no padrão de consumo, considerando a finitude dos recursos naturais utilizados de maneira inadequada (LEITE; AWAD, 2012, e AGOPYAN; JOHN, 2011). Apontase o consumo de energia oriundo de fontes não renováveis, como um dos desencadeadores deste problema.
Leite e Awad, já em 2012, apontavam que dois terços do consumo mundial de energia advinham das cidades, e os edifícios eram os responsáveis por, pelo menos, $40 \%$ da energia utilizada. As construções, além de representarem um dos setores de maior consumo de energia mundial, foram responsáveis por 39\% das emissões de carbono (UN ENVIRONMENT, 2017).

No contexto nacional, a classificação realizada pelo Anuário Estatístico de Energia Elétrica (2017) indicou o Brasil como o sétimo país com maior consumo de eletricidade do mundo em 2014. Segundo o Balanço Energético Nacional (EPE, 2019) - ano base de 2018 -, o setor residencial foi o que mais contribuiu para o aumento no consumo de energia do país. O mesmo estudo apontou que o total de emissões de $\mathrm{CO}_{2}$ associadas à matriz energética brasileira, nas residências, ficou atrás apenas do setor de transportes e das indústrias.

Salienta-se que o impacto energético que as edificações causam é uma preocupação dos setores públi- 
cos e privados em escala global, que agem pautados pelos resultados de pesquisas específicas da área para a proposição de diretrizes e tecnologias voltadas para os componentes da edificação. Pesquisadores e órgãos específicos, governamentais ou não, concentram esforços para reduzir o consumo de energia nas edificações, seja por meio de ações para adaptações nas construções já existentes, seja na definição de diretrizes específicas para novos edifícios.

Observa-se que, de acordo com as Nações Unidas (UN, 2018), até $2050,68 \%$ da população mundial poderá estar localizada em centros urbanos. Este crescimento ocasiona a maior ocupação do solo urbano por meio da verticalização, influenciando nas características dos ambientes, especialmente nos países tropicais que dependem da ventilação natural para obtenção de conforto. Estas novas configurações espaciais do ambiente construído ampliam a necessidade, em grande parte, do uso de sistemas artificiais de condicionamento.

A expansão cada vez maior das áreas urbanizadas e a mudança de hábitos em função da redução nos custos de equipamentos elétricos, como os condicionares de ar, leva a um maior consumo energético no setor da construção civil. Destaca-se que o desenvolvimento rápido das construções em países como a China e a Índia tem feito o consumo de energia mundial crescer ainda mais (WBCSD, 2007).
Dados de 2018 da Agência de Energia Internacional (IEAc, 2018) mostram que a quantidade de domicílios equipados com ar condicionado na China é de $60 \%$, o que corresponde a 643 milhões de unidades, com expectativa de se chegar a 1 bilhão e 419 milhões de unidades em 2050. A Índia apresenta apenas $5 \%$, com 36 milhões de equipamentos, porém sua projeção é de 1 bilhão e 144 milhões de unidades em 2050, quando cerca de 2/3 das famílias do mundo devem ter um ar condicionado. China, Índia e Indonésia juntos representarão metade do número total de aparelhos.

Como a demanda de energia para resfriamento (IEAa, 2019) é o tipo de consumo que mais cresce nos edifícios, as vendas de equipamentos para refrigeração estão subindo 3 vezes mais rápido do que as melhorias de eficiência dos aparelhos, salientando que a cada segundo são vendidos 10 condicionadores de ar, o que deve acontecer pelos próximos 30 anos. Com quase 1,6 bilhão de unidades de ar condicionado em operação global, o resfriamento representou, em 2017, 20\% do uso de eletricidade do setor de edifícios. O número de condicionadores de ar aumentou $50 \%$ na última década, transformando o resfriamento de espaço, no principal condutor da nova demanda de energia nos edifícios.

O uso destes sistemas de condicionamento de ar, requer potencializar soluções tecnológicas e construtivas para minimizar os efeitos do desconforto ambiental, considerando que o usuário permanece 
de $80 \%$ a $90 \%$ do dia no interior dos edifícios (RUPP; VÁSQUEZ; LAMBERTS, 2015).

A realidade do século $X X I$, com o grande consumo de aparelhos de ar condicionado, permitiu liberdade criativa dos projetistas para propor edificações, nas quais o clima interno é dissociado do externo, resultando no isolamento do usuário em edifícios composto por uma envolvente com propriedades térmicas incoerentes com as condições climáticas, resultando em espaços somente ocupados com a presença da climatização artificial (ROAF; CRICHTON; NICOL, 2009).

Salienta-se que o baixo desempenho da edificação induz o usuário à utilização de equipamentos para a obtenção do nível de conforto adequado, o que envolve consumo de energia (ROAF; CRICHTON; NICOL, 2009). Ao contrário, para o WBCSD (2007), a eficiência da edificação envolve a redução do consumo de energia para níveis aceitáveis de conforto, qualidade do ar e outros requisitos ocupacionais, incluindo a energia incorporada nos materiais e na operação das edificações.

Assim, usar as abordagens passivas, como a ventilação natural, é de grande importância uma vez que contribui para mitigar a dependência de recursos energéticos, fósseis ou não, e, ao mesmo tempo, fornecem oportunidades para diminuir as emissões de gases de efeito estufa de forma direta ou indireta. Ademais, as abordagens passivas são um meio eficaz para auxiliar na melhoria da qualidade do ar interior, podendo ainda auxiliar nas condições para o alcance do conforto térmico (SALEEM et al., 2016). Sorgato, Melo e Lamberts (2016) ressaltam que, em países com clima tropical, a estratégia de ventilação natural, quando associada ao arrefecimento artificial, contribui de forma relevante para reduzir o consumo de energia e garantir o conforto dos usuários.

Neste contexto, sabe-se que as janelas têm um impacto considerável e, muitas vezes, complexo no consumo de energia no ambiente interno, tendo papel central nesse desenvolvimento (SKARNING; HVIID; SVENDSEN, 2016). As janelas definem o nível de habitabilidade do edifício e, por interferirem no controle de luz e vento, contribuem para a eficiência energética (NICO RODRIGUES et al., 2015).

Observa-se, nos dias atuais, pouca utilização de estratégias pautadas nos princípios bioclimáticos para se obter ambientes termicamente confortáveis e com baixo consumo de energia (NICO RODRIGUES et al., 2015). As estratégias adequadas potencializam soluções construtivas energeticamente eficientes, utilizando a ventilação natural como fator de obtenção do conforto térmico, minimizando o condicionamento de ar (ROAF; CRICHTON; NICOL, 2009). Gonçalves e Bode (2015) mencionam que os edifícios que se utilizam dessas estratégias, ao invés de se isolarem do clima externo, apresentam uma relação com o espaço externo nos diferen- 
tes contextos climáticos

Em edificações localizadas em regiões tropicais, a ventilação natural é um dos fatores que influenciam no desempenho energético quando proporcionada pelo uso do modelo adequado de janela, gerando uma redução de $36,44 \%$ de $\mathrm{kWh}$ no consumo de energia (COSTALONGA et al., 2017). Soma-se a isso que as janelas representam o componente da edificação que possui menor isolamento térmico da envoltória e é, assim, um elemento chave no alcance do menor consumo de energia em edifícios (NICO RODRIGUES et al., 2015).

Dessa forma, as janelas surgem como elemento capaz de minimizar o cenário da crise energética, permitindo às edificações obterem características eficientes. As janelas corretamente isoladas têm grande potencial para reduzir o consumo de energia em novos edifícios e em instalações e retrofits estruturais, maximizando ou minimizando os ganhos solares, e podem reduzir significativamente a demanda de aquecimento ou resfriamento, especialmente para edifícios com muito vidro (IEAb, 2019).

As janelas são, portanto, centrais para o desenvolvimento de residências habitáveis com baixo consumo. Vários estudos indicaram que o efeito das janelas no consumo de energia, pode mudar significativamente com a melhoria dos níveis de isola- mento do edifício (SKARNING; HVIID; SVENDSEN, 2015).

Diante desse contexto, questiona-se se há uma investigação na literatura científica mundial, envolvendo a utilização de janelas para a redução do consumo energético atrelado às edificações. $E$, existindo essa investigação, quais aspectos estão sendo estudados e quais países têm desenvolvido essas pesquisas.

Para tanto, este estudo faz uma revisão sistemática de literatura relacionando o elemento janela ao consumo de energia. Com isso, o objetivo foi identificar quais foram as características da janela mais abordadas na produção científica internacional, com ênfase na obtenção do desempenho energético da edificação e o conforto térmico.

\section{Procedimentos Metodológicos}

Foi realizado um estudo teórico de natureza qualiquantitativa, cujo desenvolvimento se deu por meio de uma revisão sistemática da literatura científica. Os procedimentos metodológicos basearam-se nos estudos desenvolvidos por Rupp, Vásquez e Lamberts (2015) e França e Mariani (2017), que definem os critérios para a escolha das bases de dados eletrônicas, as estratégias de pesquisa e os descritores de busca, como também os parâmetros de relevância e os procedimentos para análise do conteúdo e 


\begin{tabular}{|c|c|c|c|}
\hline Termos & Buscador & Combinações & $\begin{array}{c}\text { Bases de } \\
\text { Dados }\end{array}$ \\
\hline & & $\begin{array}{l}\text { 1. "Building OR architecture AND } \\
\text { window" }\end{array}$ & \\
\hline Building & & $\begin{array}{l}\text { 2. "Building OR architecture AND } \\
\text { window AND efficient" }\end{array}$ & \\
\hline Architecture & & $\begin{array}{l}\text { 3. "Building OR architecture AND } \\
\text { window AND design" }\end{array}$ & \\
\hline Window & "aspas" & $\begin{array}{l}\text { 4. "Building OR architecture AND } \\
\text { window AND energy" }\end{array}$ & Scopus \\
\hline Design & OR & $\begin{array}{l}\text { 5. "Building OR architecture AND } \\
\text { window AND efficient AND design" }\end{array}$ & $\begin{array}{l}\text { Web of } \\
\text { Science }\end{array}$ \\
\hline Energy & AND & $\begin{array}{l}\text { 6. "Building OR architecture AND } \\
\text { window AND efficient AND energy" }\end{array}$ & Science Direct \\
\hline $\begin{array}{l}\text { Energy } \\
\text { Consumption }\end{array}$ & & $\begin{array}{l}\text { 7. "Building OR architecture AND } \\
\text { window AND design AND energy" }\end{array}$ & \\
\hline \multirow[t]{2}{*}{ Efficient } & & $\begin{array}{l}\text { 8. "Building OR architecture AND } \\
\text { window AND design AND energy } \\
\text { AND efficient" }\end{array}$ & \\
\hline & & 9. "Window AND energy consumption" & \\
\hline
\end{tabular}

Figura 1. Combinações possíveis entre os descritores de busca. Fonte: Acervo dos Autores, 2019.

sua sistematização. Estes procedimentos foram adaptados para o objetivo desta pesquisa.

O recorte temporal não foi definido, por entender que a temática deveria abranger desde a primeira publicação até o ano de 2018, quando se finalizou o período de busca. Foram feitas pesquisas somente em artigos, com aplicação de filtros de busca na área e no título.

A definição das bases de dados, baseou-se na metodologia proposta por Rupp, Vásquez e Lamberts
(2015), que utilizaram a Scopus, a Web of Science, a Science Direct e o Google Scholar. França e Mariani (2017) colocam que o Google Scholar é uma ferramenta de busca que efetua pesquisas simultaneamente em diversas fontes, não podendo, portanto, ser considerada uma base de dados. Optou-se, então, por não utilizar o Google Scholar, realizando a pesquisa na Scopus e na Web of Science, que correspondem às fontes primárias da produção científica, e na Science Direct, por ser uma base com textos completos, mesmo possuindo uma amplitude menor, cuja importância está nas publicações na área de arquitetura e engenharia.

A temática para a determinação dos descritores de busca considerou a relação entre as janelas e o consumo de energia na edificação. Para a definição do descritor de busca na língua inglesa, utilizou-se o Sistema da Fundação Biblioteca Nacional (2018) buscando "autoridades" pelo "termo tópico", na aba Library of Congress, definindo o termo na linguagem científica, segundo indicação de França e Mariani (2017).

Os resultados dos descritores de busca no Sistema da Fundação Biblioteca Nacional foram os seguintes: Building (construção), Architecture (arquitetura), Window (janela), Design (projeto), Energy (energia), Energy Consumption (consumo de energia) e Efficient (eficiente). As buscas foram realizadas combinando os termos, de acordo com os recursos de cada buscador, tais como truncagem, aspas e ope 
rador booleano, conforme Figura 1.

Cada base de dados possui uma busca por relevância de acordo com critérios específicos. $\mathrm{Na}$ Web of Science (2018), a busca por relevância classifica os registros em ordem decrescente, referente à quantidade de termos encontrados em cada um. Os registros consideraram os seguintes campos: Título, Resumo, Palavras-chave e Palavras-chave Plus; sendo que os campos de Título e Palavras-chave têm maior peso do que os demais. Para a Elsevier (Scopus, 2018), fornecedora das bases Scopus e Science Direct, a relevância é obtida por meio dos seguintes fatores que contribuem na pontuação da classificação: número de acessos; quão significativa é uma palavra? ; onde a palavra é encontrada? ; posição no documento; proximidade; e completude.

Isso significa que todos esses fatores foram considerados pelos algoritmos de busca das bases de dados. Não se escolheu nenhum deles para ter maior peso na pesquisa, mantendo-se as configurações padrões de busca por relevância das bases.

Os resultados das 9 combinações de buscas, nas 3 bases de dados, foram organizados por relevância (de acordo com os critérios estabelecidos por cada base de dados) para verificar quais apresentavam, entre seus principais resultados, apenas artigos relacionados ao objeto de estudo. Analisa- ram-se os títulos e os resumos dos 10 primeiros artigos de cada descritor, ordenando-os por relevância (em ordem decrescente) em cada uma das 3 bases de dados.

A análise identificou o descritor que obteve os resultados que melhor se relacionam ao objeto de estudo, excluindo as demais combinações, cujos resultados mais relevantes fugiam do tema da pesquisa. Após a definição do descritor, foram organizados os resultados obtidos nas 3 bases de dados utilizadas no software Mendeley, para eliminar as duplicações e facilitar a categorização dos artigos. Os títulos e os resumos dos artigos foram analisados e utilizou-se o critério de descartar, para aqueles cujo tema não era o objeto de estudo ou; que não apresentava o elemento janela como um dos principais assuntos ou; cujos resultados obtidos não eram relacionados às janelas. Em alguns casos, foi necessária a leitura do artigo devido à carência de informações nos resumos.

Para realizar a sistematização dos resultados, os artigos foram analisados e classificados de acordo com o seu objeto de estudo, sendo apresentados de forma quantitativa, separados por ano e por país, com a identificação em mapa, dos países que realizaram pesquisas e seus quantitativos. Para uma análise mais detalhada do quantitativo de produção, relacionou-se o resultado aos eventos políticos e econômicos que tenham impactado nas produções acadêmicas desenvolvidas. 


\begin{tabular}{|c|c|c|c|c|}
\hline Combinaçäo & $\begin{array}{l}\text { Termo de pesquisa na } \\
\text { Scopus }\end{array}$ & $\begin{array}{l}\text { Termo de pesquisa na } \\
\text { Web of Science }\end{array}$ & $\begin{array}{l}\text { Termo de pesquisa na } \\
\text { Science Direct }\end{array}$ & Total de resultados \\
\hline $\begin{array}{l}\text { Building OR architecture } \\
\text { AND window }\end{array}$ & $\begin{array}{l}\text { (building } O R \text { architecture) } \\
\text { AND window }\end{array}$ & $\begin{array}{l}\text { TS'=(building OR } \\
\text { architecture) AND } \\
\text { TS=window }\end{array}$ & $\begin{array}{l}\text { (building OR architecture) } \\
\text { AND window }\end{array}$ & $\begin{array}{l}\text { Scopus }=7.429 \\
\text { Web of Science }=2.377 \\
\text { Science Direct }=10.181 \\
\text { Total }=19.987\end{array}$ \\
\hline $\begin{array}{l}\text { Building } O R \text { architecture } \\
\text { AND window AND } \\
\text { efficient }\end{array}$ & $\begin{array}{l}\text { (building OR } \\
\text { architecture) AND } \\
\text { window efficien* }\end{array}$ & $\begin{array}{l}\text { TS=(building OR } \\
\text { architecture) AND } \\
\text { TS=window AND } \\
\text { TS=efficien* }\end{array}$ & $\begin{array}{l}\text { (building OR } \\
\text { architecture) AND } \\
\text { window AND efficient }\end{array}$ & $\begin{array}{l}\text { Scopus }=1.574 \\
\text { Web of Science }=585 \\
\text { Science Direct }=8.399 \\
\text { Total }=10.558\end{array}$ \\
\hline $\begin{array}{l}\text { Building OR architecture } \\
\text { AND window AND design }\end{array}$ & $\begin{array}{l}\text { (building OR } \\
\text { architecture) AND } \\
\text { window AND design }\end{array}$ & $\begin{array}{l}\text { TS=(building OR } \\
\text { architecture) AND } \\
\text { TS=window AND } \\
\text { TS=design }\end{array}$ & $\begin{array}{l}\text { (building OR } \\
\text { architecture) AND } \\
\text { window AND design }\end{array}$ & $\begin{array}{l}\text { Scopus }=2.523 \\
\text { Web of Science }=945 \\
\text { Science Direct }=9.871 \\
\text { Total }=13.339\end{array}$ \\
\hline $\begin{array}{l}\text { Building } O R \text { architecture } \\
\text { AND window AND energy }\end{array}$ & $\begin{array}{l}\text { (building OR } \\
\text { architecture) AND } \\
\text { window AND energy }\end{array}$ & $\begin{array}{l}\text { TS=(building OR } \\
\text { architecture) AND } \\
\text { TS=window AND } \\
\text { TS=energy }\end{array}$ & $\begin{array}{l}\text { (building OR } \\
\text { architecture) AND } \\
\text { window AND energy }\end{array}$ & $\begin{array}{l}\text { Scopus }=2.655 \\
\text { Web of Science }=1.254 \\
\text { Science Direct }=10.466 \\
\text { Total }=14.375\end{array}$ \\
\hline $\begin{array}{l}\text { Building } O R \text { architecture } \\
\text { AND window AND } \\
\text { efficient AND design }\end{array}$ & $\begin{array}{l}\text { (building OR } \\
\text { architecture) AND } \\
\text { window AND efficien* } \\
\text { AND design }\end{array}$ & $\begin{array}{l}\text { TS=(building OR } \\
\text { architecture) AND } \\
\text { TS=window AND } \\
\text { TS=efficien* AND } \\
\text { TS=design }\end{array}$ & $\begin{array}{l}\text { (building OR } \\
\text { architecture) AND } \\
\text { window AND efficient } \\
\text { AND design }\end{array}$ & $\begin{array}{l}\text { Scopus }=631 \\
\text { Web of Science }=282 \\
\text { Science Direct }=7.363 \\
\text { Total }=8.276\end{array}$ \\
\hline $\begin{array}{l}\text { Building } O R \text { architecture } \\
\text { AND window AND } \\
\text { efficient AND energy }\end{array}$ & $\begin{array}{l}\text { (building OR } \\
\text { architecture) AND } \\
\text { window AND efficien* } \\
\text { AND energy }\end{array}$ & $\begin{array}{l}\text { TS=(building OR } \\
\text { architecture) AND } \\
\text { TS=window AND } \\
\text { TS=efficien* AND } \\
\text { TS=energy }\end{array}$ & $\begin{array}{l}\text { (building OR } \\
\text { architecture) AND } \\
\text { window AND efficient } \\
\text { AND energy }\end{array}$ & $\begin{array}{l}\text { Scopus }=1.101 \\
\text { Web of Science }=505 \\
\text { Science Direct }=8.129 \\
\text { Total }=9.735\end{array}$ \\
\hline $\begin{array}{l}\text { Building } O R \text { architecture } \\
\text { AND window AND design } \\
\text { AND energy }\end{array}$ & $\begin{array}{l}\text { (building OR } \\
\text { architecture) AND } \\
\text { window AND design AND } \\
\text { energy }\end{array}$ & $\begin{array}{l}\text { TS=(building OR } \\
\text { architecture) AND } \\
\text { TS=window AND } \\
\text { TS=design AND } \\
\text { TS=energy }\end{array}$ & $\begin{array}{l}\text { (building OR } \\
\text { architecture) AND } \\
\text { window AND design AND } \\
\text { energy }\end{array}$ & $\begin{array}{l}\text { Scopus }=1.015 \\
\text { Web of Science }=560 \\
\text { Science Direct }=9.120 \\
\text { Total }=10.695\end{array}$ \\
\hline $\begin{array}{l}\text { Building } O R \text { architecture } \\
\text { AND window AND design } \\
\text { AND energy AND } \\
\text { efficient }\end{array}$ & $\begin{array}{l}\text { (building OR } \\
\text { architecture) AND } \\
\text { window AND design AND } \\
\text { energy AND efficien* }\end{array}$ & $\begin{array}{l}\text { TS=(building OR } \\
\text { architecture) AND } \\
\text { TS=window AND } \\
\text { TS=design AND } \\
\text { TS=energy AND } \\
\text { TS=efficien* }\end{array}$ & $\begin{array}{l}\text { (building OR } \\
\text { architecture) AND } \\
\text { window AND design AND } \\
\text { energy AND efficient }\end{array}$ & $\begin{array}{l}\text { Scopus }=474 \\
\text { Web of Science }=254 \\
\text { Science Direct }=7.713 \\
\text { Total }=8.441\end{array}$ \\
\hline $\begin{array}{l}\text { Window AND energy } \\
\text { consumption }\end{array}$ & $\begin{array}{l}\text { window AND "energy } \\
\text { consumption" }\end{array}$ & $\begin{array}{l}\text { TS=window AND } \\
\text { TS="energy } \\
\text { consumption" }\end{array}$ & $\begin{array}{l}\text { window AND "energy } \\
\text { consumption" }\end{array}$ & $\begin{array}{l}\text { Scopus }=1.051 \\
\text { Web of Science }=481 \\
\text { Science Direct }=672 \\
\text { Total }=2.204\end{array}$ \\
\hline
\end{tabular}

\section{TS=tópico.}

Figura 2. Resultados das buscas com os termos. Fonte: Acervo dos Autores, 2018.

\section{Resultados e Discussões}

Os dados obtidos proporcionaram um panorama das pesquisas relacionadas às janelas e à sua influência no consumo de energia. Os resultados foram referentes aos critérios: busca por termos; classificação por relevância de cada base; e sistematização de conteúdo (por termo; por pais e ano de publicação).

A. Busca por termos: Os resultados obtidos por cada buscador estão apresentados separadamente na figura 2.

B. Critérios de relevância de cada base de dados: após a classificação dos artigos por relevância em cada base de dados e a análise dos 10 principais resultados das 9 buscas, nas 3 bases de dados, identificou-se, como mostram as figuras 3 a 5 , que a combinação das palavras Window (janela) e Energy Consumption (consumo de energia) obteve os resultados que melhor se relacionam ao objeto de estudo. As demais combinações apresentavam, entre seus principais resultados, artigos que não contemplavam o objeto de estudo. 
Edna Aparecida Nico Rodrigues e Victor Moura Bussolotti O elemento janela versus desempenho e conforto térmico: uma sistematização de literatura

\begin{tabular}{|c|c|c|c|}
\hline \multicolumn{4}{|c|}{ Base de dados: Scopus } \\
\hline & Título do Artigo & Autores & $\begin{array}{l}\text { Ano de } \\
\text { Publicacão }\end{array}$ \\
\hline 1 & $\begin{array}{l}\text { Energy consumption in buildings: A correlation for the } \\
\text { influence of window to wall ratio and window } \\
\text { orientation in Tripoli, Libya }\end{array}$ & $\begin{array}{l}\text { Alghoul, S.K. } \\
\text { Rijabo, H.G. } \\
\text { Mashena, M.E. }\end{array}$ & 2017 \\
\hline 2 & $\begin{array}{l}\text { Technoeconomic assessment of the impact of window } \\
\text { shading retrofits on the heating and cooling energy } \\
\text { consumption and GHG emissions of the Canadian } \\
\text { housing stock }\end{array}$ & $\begin{array}{l}\text { Nikoofard, S. Ismet } \\
\text { Ugursal, V. } \\
\text { Beausoleil-Morrison, I. }\end{array}$ & 2014 \\
\hline 3 & $\begin{array}{l}\text { Economic growth and energy consumption causal } \\
\text { nexus viewed through a bootstrap rolling window }\end{array}$ & $\begin{array}{l}\text { Balcilar, M. } \\
\text { Ozdemir, Z.A. } \\
\text { Arslanturk, Y. }\end{array}$ & 2010 \\
\hline 4 & $\begin{array}{l}\text { Lighting performance and electrical energy } \\
\text { consumption of a virtual window prototype }\end{array}$ & $\begin{array}{l}\text { Mangkuto, R.A. } \\
\text { Wang, S. } \\
\text { Meerbeek, B.W. } \\
\text { Aries, M.B.C. } \\
\text { van Loenen. E.J. }\end{array}$ & 2014 \\
\hline 5 & $\begin{array}{l}\text { Contrastive analyses on annual energy consumption } \\
\text { characteristics and the influence mechanism between } \\
\text { new and old residential buildings in Shanghai, China, } \\
\text { by the statistical methods }\end{array}$ & $\begin{array}{l}\text { Chen, S. Yoshino, } \\
\text { H. Levine, M.D. } \\
\text { Li, Z. }\end{array}$ & 2009 \\
\hline 6 & $\begin{array}{l}\text { Research on the influence of external window on } \\
\text { energy consumption of residential building in the hot } \\
\text { summer and cold winter zone }\end{array}$ & $\begin{array}{l}\text { Li, Z.-R. Zhang, H.- } \\
\text { D. Tang, Z. Lin, X. }\end{array}$ & 2007 \\
\hline 7 & $\begin{array}{l}\text { Does window-to-wall ratio have a significant effect on } \\
\text { the energy consumption of uildings? A parametric } \\
\text { analysis in Italian climate conditions }\end{array}$ & $\begin{array}{l}\text { Marino, C. Nucara, } \\
\text { A. Pietrafesa, M. }\end{array}$ & 2017 \\
\hline 8 & $\begin{array}{l}\text { Experimental and theoretical study on the effect of } \\
\text { window films on building energy consumption }\end{array}$ & $\begin{array}{l}\text { Li, C. Tan, J. Chow, } \\
\text { T.-T. Qiu, Z. }\end{array}$ & 2015 \\
\hline 9 & $\begin{array}{l}\text { Impact of vindow films on the overall energy } \\
\text { consumption of existing UK hotel buildings }\end{array}$ & $\begin{array}{l}\text { Bahadori-Jahromi, } \\
\text { A. Rotimi, A. } \\
\text { Mylona, A. Godfrey, } \\
\text { P. Cook, D. }\end{array}$ & 2017 \\
\hline 10 & $\begin{array}{l}\text { Reduction of energy consumption by AC due to air } \\
\text { tightness and ventilation strategy in residences in hot } \\
\text { and humid climates }\end{array}$ & $\begin{array}{l}\text { Uno, T. Hokoi, S. } \\
\text { Ekasivi, S.N.N. } \\
\text { Abdul Majid. N.H. }\end{array}$ & 2012 \\
\hline
\end{tabular}

Figura 3. Os 10 artigos selecionados pelos critérios de relevância na base Scopus. Fonte: Acervo dos Autores, 2018.

\begin{tabular}{|c|c|c|c|}
\hline \multicolumn{4}{|c|}{ Base de dados: Web of Science } \\
\hline & Título do Artigo & Autores & $\begin{array}{l}\text { Ano de } \\
\text { Publicação }\end{array}$ \\
\hline 1 & $\begin{array}{l}\text { Energy consumption in buildings: A correlation for the } \\
\text { influence of window to wall ratio and window } \\
\text { orientation in Tripoli, Libya }\end{array}$ & $\begin{array}{l}\text { Alghoul, Samah K.; } \\
\text { Rijabo, Hassan G.; } \\
\text { Mashena, Mohamed E. }\end{array}$ & 2017 \\
\hline 2 & $\begin{array}{l}\text { Impact of Window Films on the Overall Energy } \\
\text { Consumption of Existing UK Hotel Buildings }\end{array}$ & $\begin{array}{l}\text { Bahadori-Jahromi, Ali; } \\
\text { Rotimi, Abdulazeez; } \\
\text { Mylona, Anastasia; et al. }\end{array}$ & 2017 \\
\hline 3 & $\begin{array}{l}\text { A study on the proposes of energy analysis indicator } \\
\text { by the vindow elements of office buildings in Korea }\end{array}$ & $\begin{array}{l}\text { Kim, Seok-Hyun; Kim, } \\
\text { Sun-Sook; Kim, Kwang- } \\
\text { Woo; et al. }\end{array}$ & 2014 \\
\hline 4 & $\begin{array}{l}\text { Theoretical discussions of perfect window, ideal near } \\
\text { infrared solar spectrum regulating window and current } \\
\text { thermochromic window }\end{array}$ & $\begin{array}{l}\text { Ye, Hong; Meng, } \\
\text { Xianchun; Xu, Bin }\end{array}$ & 2012 \\
\hline 5 & $\begin{array}{l}\text { Window operation and impacts on building energy } \\
\text { consumption }\end{array}$ & $\begin{array}{l}\text { Wang, Liping; } \\
\text { Greenberq. Steve }\end{array}$ & 2015 \\
\hline 6 & $\begin{array}{l}\text { Dynamic modeling of potentially conflicting energy } \\
\text { reduction strategies for residential structures in semi- } \\
\text { arid climates }\end{array}$ & $\begin{array}{l}\text { Hester, Nathan; Li, Ke; } \\
\text { Schramski, John R.; et al. }\end{array}$ & 2012 \\
\hline 7 & $\begin{array}{l}\text { Optimization of window size design for detached } \\
\text { house using Trnsys Simulations and genetic algorithm }\end{array}$ & $\begin{array}{l}\text { Ferdyn-Grygierek, } \\
\text { Joanna; Grygierek, } \\
\text { Krzysztof }\end{array}$ & 2017 \\
\hline 8 & $\begin{array}{l}\text { Reduced energy consumption and enhanced comfort } \\
\text { with smart windows: Comparison between quasi- } \\
\text { optimal, predictive and rule-based control strategies }\end{array}$ & $\begin{array}{l}\text { Dussault, Jean-Michel; } \\
\text { Sourbron, Maarten; } \\
\text { Gosselin, Louis }\end{array}$ & 2016 \\
\hline 9 & $\begin{array}{l}\text { Technoeconomic Assessment of the Impact of } \\
\text { Window Improvements on the Heating and Cooling } \\
\text { Energy Requirement and Greenhouse Gas Emissions } \\
\text { of the Canadian Housing Stock }\end{array}$ & $\begin{array}{l}\text { Nikoofard, Sara; Ugursal, } \\
\text { V. Ismet, Beausoleil- } \\
\text { Morrison, lan }\end{array}$ & 2014 \\
\hline 10 & $\begin{array}{l}\text { Technoeconomic assessment of the impact of window } \\
\text { shading retrofits on the heating and cooling energy } \\
\text { consumption and GHG emissions of the Canadian } \\
\text { housing stock }\end{array}$ & $\begin{array}{l}\text { Nikoofard, Sara; Ugursal, } \\
\text { V. Ismet, Beausoleil- } \\
\text { Morrison, lan }\end{array}$ & 2014 \\
\hline
\end{tabular}

Figura 4. Os 10 artigos selecionados pelos critérios de relevância na base Web of Science. Fonte: Acervo dos Autores, 2018 


\begin{tabular}{|c|c|c|c|}
\hline \multicolumn{4}{|c|}{ Base de dados: Science Direct } \\
\hline & Título do Artigo & Autores & $\begin{array}{l}\text { Ano de } \\
\text { Publicação }\end{array}$ \\
\hline 1 & $\begin{array}{l}\text { Energy investigation of glazed windows containing } \\
\text { Nano-PCM in different seasons }\end{array}$ & $\begin{array}{l}\text { Dong Li, Yangyang Wu, } \\
\text { Changyu Liu, Guojun } \\
\text { Zhang, Müslüm Arıcl }\end{array}$ & 2018 \\
\hline 2 & $\begin{array}{l}\text { A multi-objective optimization methodology for } \\
\text { window design considering energy consumption, } \\
\text { thermal environment and visual performance }\end{array}$ & $\begin{array}{l}\text { Yingni Zhai, Yi Wang, } \\
\text { Yanqiu Huang, Xiaojing } \\
\text { Meng }\end{array}$ & 2018 \\
\hline 3 & $\begin{array}{l}\text { Energy consumption in buildings: A correlation for the } \\
\text { influence of window to wall ratio and window } \\
\text { orientation in Tripoli, Libya }\end{array}$ & $\begin{array}{l}\text { Samah K. Alghoul, } \\
\text { Hassan G. Rijabo, } \\
\text { Mohamed E. Mashena }\end{array}$ & 2017 \\
\hline 4 & $\begin{array}{l}\text { Integrated semi-transparent cadmium telluride } \\
\text { photovoltaic glazing into windows: Energy and } \\
\text { daylight performance for different architecture designs }\end{array}$ & $\begin{array}{l}\text { Yanyi Sun, Katie Shanks, } \\
\text { Hasan Baig, Wei Zhang, } \\
\text {... Yupeng Wu }\end{array}$ & 2018 \\
\hline 5 & $\begin{array}{l}\text { Window-opening behavior in Chinese residential } \\
\text { buildings across different climate zones }\end{array}$ & $\begin{array}{l}\text { Dayi Lai, Susu Jia, Yue } \\
\text { Qi, Juniie Liu }\end{array}$ & 2018 \\
\hline 6 & $\begin{array}{l}\text { The Impact of Window Configuration on the Overall } \\
\text { Building Energy Consumption under Specific Climate } \\
\text { Conditions }\end{array}$ & $\begin{array}{l}\text { Zekraoui Djamel, } \\
\text { Zemmouri Noureddine }\end{array}$ & 2017 \\
\hline 7 & $\begin{array}{l}\text { Simulation research on the dynamic thermal } \\
\text { performance of a novel triple-glazed window filled with } \\
\text { PCM }\end{array}$ & $\begin{array}{l}\text { Shuhong Li, Kaikai Zou, } \\
\text { Gaofeng Sun, Xiaosong } \\
\text { Zhang }\end{array}$ & 2018 \\
\hline 8 & $\begin{array}{l}\text { Analysis of correlation between actual heating energy } \\
\text { consumption and building physics, heating system, } \\
\text { and room position using data mining approach }\end{array}$ & $\begin{array}{l}\text { Hao Zhou, Borong Lin, } \\
\text { Jianqiang Qi, Lihong } \\
\text { Zheng, Zhongchen } \\
\text { Zhang }\end{array}$ & 2018 \\
\hline 9 & $\begin{array}{l}\text { Assessment of the efficiency of window integrated } \\
\text { CdTe based semi-transparent photovoltaic module }\end{array}$ & $\begin{array}{l}\text { Sankar Barman, Amartya } \\
\text { Chowdhury, Sanjay } \\
\text { Mathur, Jyotirmay Mathur }\end{array}$ & 2018 \\
\hline 10 & $\begin{array}{l}\text { Evaluation of the thermal and optical performance of } \\
\text { thermochromic windows for office buildings in China }\end{array}$ & $\begin{array}{l}\text { Runqi Liang, Yanyi Sun, } \\
\text { Marina Aburas, Robin } \\
\text { Wilson, Yupeng Wu }\end{array}$ & 2018 \\
\hline
\end{tabular}

usjt • arq.urb • número 28 | maio-agosto de 2020
Para o descritor de busca selecionado, combinação das palavras Window e Energy Consumption, foram obtidos 2.204 artigos, sendo 672 na Science Direct, 1.051 na Scopus e 481 na Web of Science. Esses artigos foram organizados pelo Software Mendeley, que eliminou as duplicações, resultando em 1.412 artigos. Dos 1.412 analisados, foram descartados os artigos cujo tema não era o objeto de estudo ou; que não apresentava o elemento janela como um dos principais assuntos ou; cujos resultados obtidos não eram relacionados às janelas, totalizando 744 artigos que atenderam aos critérios estabelecidos.

C. Sistematização do conteúdo: caracterizou o assunto abordado no artigo, bem como os países envolvidos e as datas de publicação. Para a caracterização do assunto abordado, efetuou-se a organização dos resultados, dividindo-os em duas categorias: as com variáveis dos elementos e posicionamento da janela; e as com os fatores que influenciam no desempenho da edificação.

- Variáveis dos elementos e posicionamento da janela: Dimensão (relação entre o tamanho da janela e a parede ou entre o ambiente que ela atende e o dimensionamento recomendado); Local (análise das orientações do edifício e a posição da janela em relação à fachada ou ao ambiente); Elementos de Sombreamento (dispositivos, tipologias e materiais recomendados e o tipo de sistema empregado); Propriedades do Vidro (estudos de sistemas de en- 


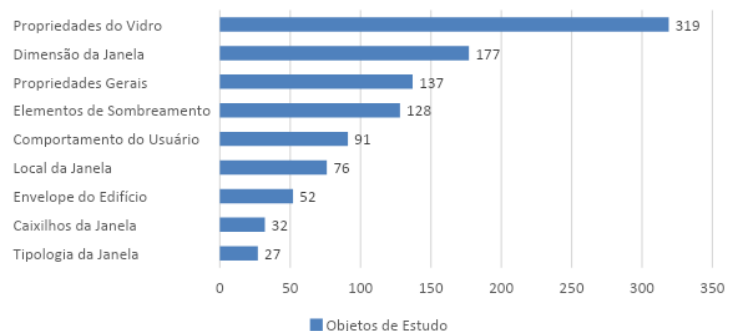

Figura 6. Variáveis mais observadas na categoria elementos e posicionamento das janelas. Fonte: Acervo dos Autores 2019.

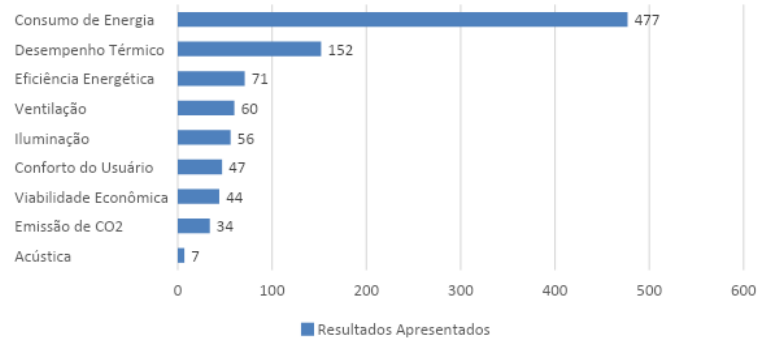

Figura 7. Fatores mais observados na categoria fatores que influenciam o desempenho do edifício. Fonte: Autor, 2019.

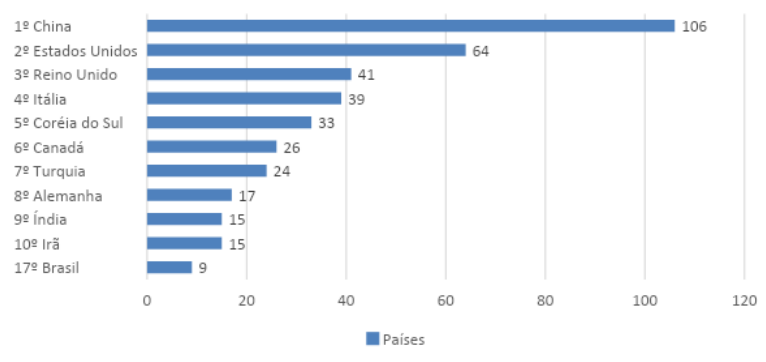

Figura 8. Países de publicação dos artigos. Fonte: Autor 2019.

usjt • arq.urb • número 28 | maio-agosto de 2020 vidraçamento, tais como vidros a vácuo, duplos, tri- forto do Usuário (satisfação do usuário com o ambiplos, com isolamento térmico, com propriedades eletrocrômicas ou placas fotovoltaicas, janelas inteligentes e os coeficientes $U$ de transmitância); Tipologia da Janela (diferentes tipologias, sistemas de abertura e operação); Caixilhos da Janela (materiais e propriedades); Propriedades gerais que analisam a esquadria, sem diferenciar seus elementos; Envelope do Edifício (elementos da fachada); e Comportamento do Usuário em relação ao uso da esquadria (frequência de abertura das janelas, seus horários e motivações).

- Fatores que influenciam o desempenho do edifício: Eficiência Energética (desempenho das janelas ou do edifício); Consumo de Energia (diminuição do consumo de energia ou o quantitativo de consumo de energia gasto pelo sistema); Desempenho Térmico (carga térmica transferida pela janela, níveis de isolamento e coeficientes de perda de calor); Emissão de $\mathrm{CO}_{2}$ (quantidade de gás necessário para a produção das esquadrias e a economia dos diversos sistemas de fenestração ao longo do ciclo de vida do material, impactos na natureza, e sua viabilidade sob a ótica ambiental); lluminação (disponibilidade de luz no ambiente e os índices de aproveitamento da luz solar); Ventilação natural (taxas de qualidade e renovação do ar no ambiente, padrões de fluxo e estanqueidade do ar); Acústica (nível de ruído no ambiente); Viabilidade Econômica (custos de instalação do sistema de janelas, viabilidade e tempo de retorno do investimento); e Con- Unido. ente e suas propriedades térmicas, acústicas e visuais).

Os artigos foram classificados dentro das categorias acima descritas, podendo receber mais de uma classificação quando tratar de mais de um elemento ou posicionamento da janela ou apresentar múltiplos fatores que influenciam o desempenho do edifício em seus resultados.

Na figura 6 foram apresentados os resultados obtidos da categoria de variáveis dos elementos e posicionamento da janela, em que a propriedade do vidro foi o fator mais investigado com 319 artigos, seguida pela dimensão da janela, com 177. O item menos estudado foi a tipologia da janela, com 27 , precedido pelos caixilhos da janela, considerando um universo de 744 artigos selecionados.

Na figura 7, os resultados apresentados da categoria de fatores que influenciam o desempenho do edifício, indicaram que o consumo de energia foi estudado em 477 artigos, seguido pelo desempenho térmico, com 152 resultados, para um total de 744 artigos.

Os países onde se localizam as universidades responsáveis pelos artigos analisados, foram computados e o resultado, representado na figura 8, aponta a China como principal produtora de conteúdo científico, seguida pelos Estados Unidos e pelo Reino 


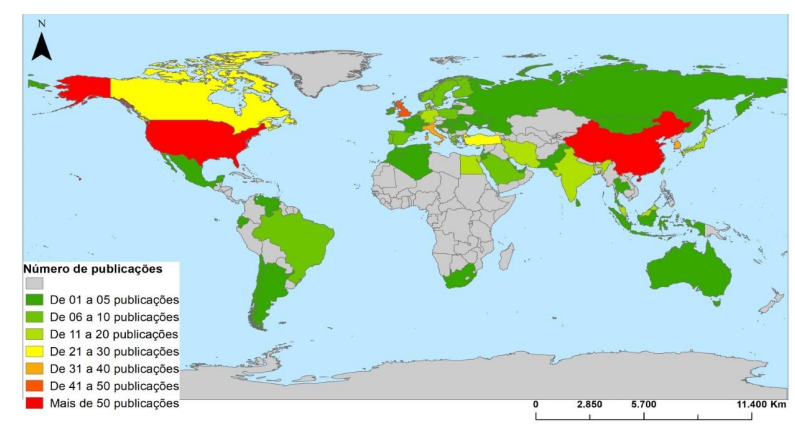

Figura 9. Distribuição geográfica das publicações dos artigos Fonte: Acervo dos Autores, 2019

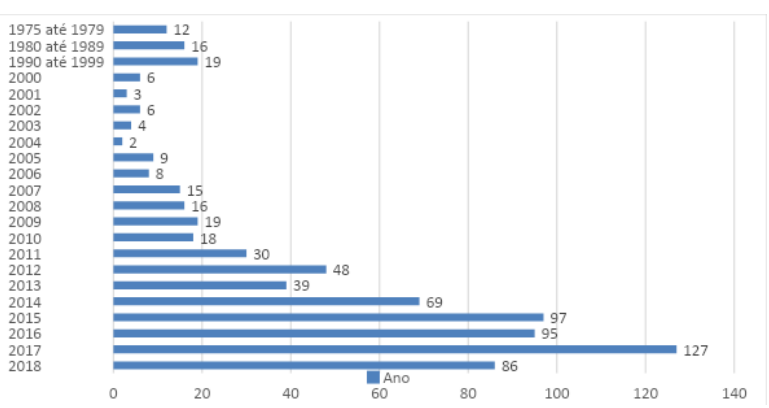

Figura 10. Ano de publicação dos artigos. Fonte: Autor, 2019.
A figura 9 ilustra a distribuição geográfica das publicações. Observa-se que América do Norte, Europa e Ásia concentram a maior parte delas. Na América do Sul, o Brasil e o Chile são as principais fontes de artigos. África e Oceania têm publicações em menor número, enquanto que a América Central não apresenta publicações.

Em relação ao ano de publicação, ilustrado na figura 10, tem-se que, dos artigos obtidos nas bases de dados, o mais antigo encontrado foi de 1975, e os mais recentes de 2018 (ano limite da busca). Constatou-se que o ano de 2017, com 127 publicações, foi o maior responsável pela quantidade de pesquisas, seguido pelo ano de 2015, com 97 artigos. No entanto, como a busca ocorreu até out/2018, este ano poderá contabilizar e ultrapassar os valores registrados no ano de 2017.

A Figura 10 demonstrou que o número de artigos publicados apresentou um crescimento ao longo dos anos. Alguns eventos de contexto político e econômico podem ter contribuído para o aumento no número de pesquisas sobre o assunto.

A recuperação após a crise econômica mundial de 2008 a 2010 é refletida no aumento de $67 \%$ no número de publicações de 2010 para 2011, saltando de 18 para 30 artigos. Na China, principal país na produção científica sobre o assunto, foi estabelecida em 2011 pelo governo, uma meta de redução da intensidade energética do país em $12 \%$, no plano quinquenal - 2011 a 2015 -, afastando a sua estrutura econômica de produtos que consomem muita energia (LI, SONG e LIU, 2014).

Nos Estados Unidos, $2^{\circ}$ colocado no ranking de países, a crise econômica de 2008 ocorreu simultaneamente à troca de presidentes, tendo Barack Obama assumido em 2009, cuja presidência marcou um momento ativo para a política climática americana. Durante sua gestão, os EUA lançaram planos de energia limpa e de redução de gases do efeito estufa, tendo atuação na conferência de Copenhague e no acordo de Paris (PISCHKE, 2018).

O Reino Unido e a Itália estão submetidos às políticas de eficiência energética da União Europeia, que visam diminuir a quantidade de energia gasta sem impedir o crescimento. Como exemplo, há a Política Climática e Energética para 2030 e a contribuição com o acordo de Paris. Há, ainda, a diretiva de eficiência energética - 2012/27/EU -, que é a principal peça de legislação da União Europeia, aplicável a todos os setores (MALINAUSKAITE et al, 2019).

A Coréia do Sul adotou a postura de reduzir os gases de efeito estufa por meio da inserção de fontes de energia renováveis, com políticas em conformidade com os acordos e conferências climáticas in-

ternacionais (KIM et al, 2018). 
O Brasil, por sua vez, é signatário da ConvençãoQuadro das Nações Unidas sobre a Mudança do Clima e membro das negociações climáticas da $\mathrm{ONU}$, implementando estratégias nacionais para reduzir as mudanças climáticas como programas e políticas de controle do desmatamento, visando o desenvolvimento de energia renovável (OCTAVIANO, PALSEV e GRUGEL, 2016).

\section{Conclusão / Considerações}

Um panorama das pesquisas que relacionam a janela, seus elementos e suas implicações no consumo de energia e no meio ambiente pôde ser observado e suas principais características foram encontradas, mapeadas e computadas, demonstrando que os procedimentos aplicados no artigo foram satisfatórios e levaram aos resultados pretendidos.

A metodologia utilizada para a seleção da literatura permitiu identificar a dificuldade existente na seleção de descritores de busca para a pesquisa de artigos relacionados às janelas, dado que o termo "window" é utilizado em outras áreas fora da construção civil. Ressalta-se que o método de seleção de bases de dados e de determinação dos descritores de busca, proposto por França e Mariani (2017), permitiu que a pesquisa fosse realizada satisfatoriamente, mostrando-se eficaz.

Observou-se que a abordagem da temática foi crescente, demonstrado pelo aumento da quantida- de de artigos publicados. Além disso, foi possível afirmar que a China, que mais causa danos ao meio ambiente, em decorrência da emissão de gases de efeito estufa para a geração de energia no setor da construção (CLIMATEWATCH, 2019), também é o líder nas pesquisas no assunto.

Quanto aos gastos com climatização, em particular, a demanda de ar-condicionado cresceu muito na China ao longo da última década, atingindo cerca de um terço do estoque global de ar-condicionado em 2017. Neste país, em 2017 havia mais de 350 milhões de unidades em uso de ar condicionado do que em 2007. Como resultado, o consumo com os aparelhos foi o equivalente a toda a eletricidade produzida no Japão e na Coréia (IEAa, 2019).

Notou-se, também, uma concentração de pesquisas relacionada às propriedades de envidraçamento, o que está atendendo às recomendações da Agência Internacional de Energia (IEAb, 2019), em seus campos prioritários de pesquisa. Porém, há poucas publicações em outras áreas, como as propriedades do caixilho da janela, ou mesmo sua tipologia. Tal fato evidencia uma busca por tecnologias recentes e inovadoras e os elementos tradicionais não possuem tal influência.

Ressalta-se que os resultados, em sua maioria, focam em soluções para o consumo de energia da edificação, sem avaliar questões importantes como a viabilidade financeira da solução, ou mesmo os 
gastos energéticos e as emissões de gases de efeito estufa.

A sistematização realizada identificou lacunas no estudo do elemento janela, aspectos como tipologia e localização das aberturas foram pouco abordados e são potencialmente importante para a eficiência energética, quando corretamente projetados. Destaca-se, também, a importância da sistematização para entender as abordagens mais recorrentes, quando relacionadas aos eventos internacionais que motivaram os questionamentos.

\section{Agradecimentos}

O presente trabalho foi realizado com apoio da Coordenação de Aperfeiçoamento de Pessoal de Nível Superior - Brasil (CAPES) - Código de Financiamento 001.

\section{Referências}

AGOPYAN, Vahan; JOHN, Vanderley M. O desafio da sustentabilidade na construção civil. São Paulo, SP: Blucher, 2011.

ALGHOUL, S. K.; RIJABO, H. G.; MASHENA, M. E. Energy consumption in buildings: A correlation for the influence of window to wall ratio and window orientation in Tripoli, Libya. Journal of Building Engineering, v. 11, p. 82-86, 2017.

ANUÁRIO ESTATÍSTICO DE ENERGIA ELÉTRICA - 2017. Disponível em: <http://www.epe.gov.br/ sites-pt/publicacoes-dados-abertos/publicacoes>. Acesso em: 16 jan. 2018.

BAHADORI-JAHROMI, A. et al. Impact of window films on the overall energy consumption of existing UK hotel buildings. Sustainability (Switzerland), v. 9, n. 5, 2017.

BALCILAR, M.; OZDEMIR, Z. A.; ARSLANTURK, $Y$. Economic growth and energy consumption causal nexus viewed through a bootstrap rolling window. Energy Economics, v. 32, n. 6, p. 13981410, 2010.

BARMAN, S. et al. Assessment of the efficiency of window integrated CdTe based semi-transparent 
photovoltaic module. Sustainable Cities and Society, v. 37, p. 250-262, 2018.

CHEN, S. et al. Contrastive analyses on annual energy consumption characteristics and the influence mechanism between new and old residential buildings in Shanghai, China, by the statistical methods. Energy and Buildings, v. 41, n. 12, p. 1347-1359, 2009.

CLIMATE WATCH DATA (CLIMATEWATCH). GIobal Historical Emissions. Disponivel em: $<$ https://www.climatewatchdata.org/ghg-emissions? regions $=$ WORLD\&sectors $=517517>$, Acesso em 21 out. 2019.

COSTALONGA, Filipe Galina; SIRTULI, Bruna Perovano; NICO-RODRIGUES, Edna Aparecida; ALVAREZ, Cristina Engel de. Economia de energia em edifícios multifamiliares utilizando diferentes modelos de janelas em Vitória-ES. XIV ENCAC I X ELACAC, p.1280-1288, 2017.

DJAMEL, Z.; NOUREDDINE, Z. The Impact of Window Configuration on the Overall Building Energy Consumption under Specific Climate Conditions. Energy Procedia, v. 115, p. 162-172, 2017.

DUSSAULT, J.-M.; SOURBRON, M.; GOSSELIN, L. Reduced energy consumption and enhanced comfort with smart windows: Comparison between quasi-optimal, predictive and rule-based control strategies. Energy and Buildings, v. 127, p. 680691, 2016.

EPE - EMPRESA DE PESQUISA ENERGÉTICA. Balanço energético nacional - 2019. Relatório Síntese / Ano Base 2018. Disponível em: <http://epe.gov.br/sites-pt/publicacoes-dados-abertos/publicacoes/PublicacoesArquivos/publicacao-377/topico-

470/Relat\%C3\%B3rio\%20S\%C3\%ADntese

\%20BEN\%202019\%20Ano\%20Base

\%202018.pdf>. Rio de Janeiro, RJ, 2019. Acesso em: 28 out. 2019.

FERDYN-GRYGIEREK, J.; GRYGIEREK, K. Optimization of window size design for detached house using trnsys simulations and genetic algorithm. Architecture civil engineering environment, v. 10, n. 4, p. 133-140, 2017.

FRANÇA, Cláudio Márcio de; MARIANI, Ana Maria Matos de. Fontes de Informação On-line. Vitória, 2017. 34 slides, color.

FUNDAÇÃO BIBLIOTECA NACIONAL. Acervo. Disponível em: <http://acervo.bn.br/sophia_web/>. Acesso em 16 jun. 2018. 
GONÇALVES, J. C. S.; BODE, K. Edifício Ambiental. São Paulo: Oficina de Textos, 2015.

HESTER, N. et al. Dynamic modeling of potentially conflicting energy reduction strategies for residential structures in semi-arid climates. Journal of Environmental Management, v. 97, n. 1, p. 148-153, 2012.

INTERNATIONAL ENERGY AGENCY (IEAa). Cooling in buildings: Tracking Clean Energy Progress. Disponível em: <https://www.iea.org/tcep/ buildings/cooling/>, Acesso em 05 jan. 2019.

INTERNATIONAL ENERGY AGENCY (IEAb). Advanced Windows. Disponível em: <https://www.iea.org/topics/innovation/buildings/gaps/advancedwindows.html>, Acesso em 30 out. 2019.

INTERNATIONAL ENERGY AGENCY (IEAc). The Future of Cooling: Opportunities for energy-efficient air conditioning. Disponível em: < https://www.iea.org/reports/the-future-of-cooling>, Acesso em 09 jun. 2020.

KIM S., LEE, H.; KIM H.; JANG, D.; KIM, H.; HUR, J.; CHO, Y.; HUR, K. Improvement in policy and proactive interconnection procedure for renewable energy expansion in South Korea. Renewable and Sustainable Energy Reviews, v. 98, p. 150-162, dez. 2018.
KIM, S.-H. et al. A study on the proposes of energy analysis indicator by the window elements of office

buildings in Korea. Energy and Buildings, v. 73, p. 153-165, 2014.

LAI, D. et al. Window-opening behavior in Chinese residential buildings across different climate zones. Building and Environment, v. 142, p. 234-243, 2018.

LEITE, Carlos; AWAD, Juliana di Cesare Marques. Cidades sustentáveis, cidades inteligentes: desenvolvimento sustentável num planeta urbano. Porto Alegre, RS: Bookman, 2012.

LI, D. et al. Energy investigation of glazed windows containing Nano-PCM in different seasons. Energy Conversion and Management, v. 172, p. 119-128, 2018a.

LI, S.; SONG, Z.; LIU, W. China's energy consumption under the global economic crisis: Decomposition ands ectoral analysis. Energy Policy, v.64, p.193-202, 2014

$\mathrm{LI}, \mathrm{S}$. et al. Simulation research on the dynamic thermal performance of a novel triple-glazed window filled with PCM. Sustainable Cities and Society, v. 40 , p. 266-273, 2018b. 
LI, Z.-R. et al. Research on the influence of external window on energy consumption of residential building in the hot summer and cold winter zone. Journal of Harbin Institute of Technology (New Series), v. 14, n. SUPPL., p. 34-37, 2007.

LIANG, R. et al. Evaluation of the thermal and optical performance of thermochromic windows for office buildings in China. Energy and Buildings, v. 176, p. 216-231, 2018.

MALINAUSKAITE, J.; JOUHARA, H.; AHMAD, L.; MILANI, M.; MONTORSI, L.; VENTURELLI, M.Energy efficiency in industry: $\mathrm{EU}$ and national policies in Italy and the UK. Energy, v. 172, p. 255-269, abr. 2019.

MANGKUTO, R. A. et al. Lighting performance and electrical energy consumption of a virtual window prototype. Applied Energy, v. 135, p. 261-273, 2014.

MARINO, C.; NUCARA, A.; PIETRAFESA, M. Does window-to-wall ratio have a significant effect on the energy consumption of buildings? A parametric analysis in Italian climate conditions. Journal of Building Engineering, v. 13, p. 169-183, 2017.
NICO-RODRIGUES, Edna Aparecida. Influência da janela no desempenho térmico de ambientes ventilados naturalmente, 202f. Tese (Doutorado em Arquitetura e Urbanismo) - Programa de PósGraduação em Arquitetura e Urbanismo, Universidade del Bio-Bio, Chile, 2015.

NICO-RODRIGUES, Edna Aparecida; ALVAREZ, Cristina Engel de; SANTO, Amábeli dell; PIDERIT, Maria Beatriz. Quando a janela define a condição de desempenho térmico em ambientes ventilados naturalmente: caso específico das edificações multifamiliares em Vitória, ES. Ambiente Construído, Porto Alegre, v. 15, n. 2, p. 7-23, abr.jun. 2015.

NIKOOFARD, S.; ISMET UGURSAL, V.; BEAUSOLEIL-MORRISON, I. Technoeconomic assessment of the impact of window shading retrofits on the heating and cooling energy consumption and GHG emissions of the Canadian housing stock. Energy and Buildings, v. 69, p. 354-366, 2014.

NIKOOFARD, S.; UGURSAL, V. I.; BEAUSOLEILMORRISON, I. Technoeconomic assessment of the impact of window improvements on the heating and cooling energy requirement and greenhouse gas emissions of the canadian housing stock. Journal of Energy Engineering, v. 140, n. 2, 2014.

OCTAVIANO, C.; PALTSEV, S.; GURGEL, Angelo C. Climate change policy in Brazil and Mexico: Re- 
sults from the MIT EPPA model. Energy Economics, v.56, p. 600-614, maio 2016.

ORGANISATION FOR ECONOMIC CO-OPERATION AND DEVELOPMENT - 2017. Disponível em: $<$ https://www.oecd.org/health/health-systems/Healthy-people-healthy-planet.pdf>. Acesso em 20 de out. 2019.

PISCHKE, Erin C.; SOLOMON, Barry D.; WELLSTEAD, Adam M. A historical analysis of US climate change policy in the Pan-American contexto. Journal of Environmental Studies and Sciences, v. 8 p. 225-232, mar. 2018.

ROAF, Susan; CRICHTON, David; NICOL, F. A adaptação de edificações e cidades às mudanças climáticas: um guia de sobrevivência para o século XXI. Porto Alegre: Bookman, 2009.

RUPP, R. F; VÁSQUEZ G. N; LAMBERTS R. A review of human thermal comfort in the built environment. Energy and Buildings. v. 105, p. 178-205, 2015.

SALEEM, A. A; BADY, M; OOKAWARA, S; RAHMAN-ABDEL, A. K. Achieving standard natural ventilation rate of dwellings in a hot-arid climate using solar chimney. Energy and Buildings, v. 133, p. 360-370, 2016.
SCOPUS. Access and use Support Center. Disponível em: <https://service-elsevier-com.ez43.periodicos.capes.gov.br/app/answers/detail/a_id/

14182/supporthub/scopus/kw/relevance/>, Acesso em 09 ou. 2018.

SKARNING, Gunnlaug Cecilie Jensen; HVIID, Christian Anker; SVENDSEN, Svend. Roadmap for improving roof and façade windows in nearly zeroenergy houses in Europe. Energy and Buildings, v. 116, p. 602-613, 2016.

SORGATO, M. J.; MELO, A. P.; LAMBERTS, R. The effect of window opening ventilation control on residential building energy consumption. Energy and Buildings, v. 133, p. 1-13, 2016.

SUN, Y. et al. Integrated semi-transparent cadmium telluride photovoltaic glazing into windows: Energy and daylight performance for different architecture designs. Applied Energy, v. 231, p. 972-984, 2018.

UN. Report of the secretary-general on the 2019 climate action summit and the way forward in 2020. [s.l: s.n.]. Disponível em: <https:// www.un.org/en/climatechange/assets/pdf/cas_report 11_dec.pdf>. 
UN ENVIRONMENT, 2017. Towards a zero-emission, efficient, and resilient buildings and construction sector. Global status report. Disponível em: $<$ https://www.worldgbc.org/sites/default/files/UNEP $\% 20188$ GABC_en $\% 20 \% 28 w e b \% 29$.pdf>. Acesso em 30 out. 2019.

UNITED NATIONS - UN DESA. Review of the World Urbanization Prospects - 2018. Disponível em:<https://www.un.org/development/desa/publications/2018-revision-of-world-urbanization-prospects.html>. Acesso em:20 de jun. 2018.

UNO, T. et al. Reduction of energy consumption by AC due to air tightness and ventilation strategy in residences in hot and humid climates. Journal of Asian Architecture and Building Engineering, v. 11, n. 2, p. 407-414, 2012.

WANG, L.; GREENBERG, S. Window operation and impacts on building energy consumption. Energy and Buildings, v. 92, p. 313-321, 2015.

WBCSD - WORLD BUSINESS COUNCIL FOR SUSTAINABLE DEVELOPMENT. Eficiência Energética em Edifícios - Realidades empresariais e oportunidades. FACTS Summary Report. 2007.
WEB OF SCIENCE. Web of Science Core Collection Help. Disponível em: <https://images.webofknowledge.com/images/help/WOS/hs_sort_options.html>, Acesso em: 09 out. 2018.

YE, H.; MENG, X.; XU, B. Theoretical discussions of perfect window, ideal near infrared solar spectrum regulating window and current thermochromic window. Energy and Buildings, v. 49, p. 164-172, 2012.

ZHAI, Y. et al. A multi-objective optimization methodology for window design considering energy consumption, thermal environment and visual performance. Renewable Energy, 2018.

ZHOU, H. et al. Analysis of correlation between actual heating energy consumption and building physics, heating system, and room position using data mining approach. Energy and Buildings, v. 166, p. 73-82, 2018. 